\title{
Oblique Wide-Angle Multi-Sector Metamaterial Absorber for Space Applications
}

\author{
Olivier Rance ${ }^{1} \mathbb{D}$, Anne Claire Lepage ${ }^{1, * \mathbb{C}}$, Xavier Begaud $\left.{ }^{1} \mathbb{(}\right)$, Kevin Elis ${ }^{2}$ and Nicolas Capet ${ }^{2} \mathbb{D}$ \\ 1 LTCI, Télécom Paris, Institut Polytechnique de Paris, 75013 Paris, France \\ 2 Centre National d'Etudes Spatiales, 31400 Toulouse, France \\ * Correspondence: anne-claire.lepage@telecom-paris.fr
}

Received: 2 July 2019; Accepted: 10 August 2019; Published: 20 August 2019

check for updates

Featured Application: A low-mass wide-angle electromagnetic absorber was designed in order to reduce interference problems arising on satellite platforms.

\begin{abstract}
This article presents the design, realization and measurement of lightweight absorbing material for space applications. The electromagnetic absorber, operating on the [2 GHz, 2.3 GHz] frequency band, is designed for oblique incidence ranging from $35^{\circ}$ to $65^{\circ}$. Wide-angle designs are demonstrated to be particularly challenging at oblique incidence and an approach consisting in dividing the surface in two different sectors with respect to the incoming angle is proposed. A specific measurement setup is presented in order to characterize this new kind of evolutive absorber. The measurement results show that the sectorial absorber achieves a reflection coefficient inferior to $-11.5 \mathrm{~dB}$, corresponding to an absorptivity above 0.965 on the frequency band [ $2 \mathrm{GHz}, 2.3 \mathrm{GHz}$ ] for both TE and TM polarizations for angles of incidence varying from $35^{\circ}$ to $65^{\circ}$.
\end{abstract}

Keywords: radar absorbing material; metamaterial; oblique incidence; RCS measurement

\section{Introduction}

Radar absorbing materials have been traditionally used for stealth purposes in the military domain. Recently, they have also become of great interest for solving electromagnetic interference problems in the aerospace domain [1,2]. In particular, satellites may comprise multiple antennas relatively close to one another, which can affect their performances. A way to mitigate the multipath crosstalk between antennas is to cover the satellite platform with an absorbing material. For this type of application, the radar absorbing structure is mounted in the vicinity of the antenna and the absorber should operate at oblique incidence for a wide range of incidence angles. This point is important as in this case, classical designs for which the maximum of absorption is obtained at normal incidence are generally insufficient. Moreover, the absorber must be ultra-light and comply with space conditions (a wide range of temperatures, geomagnetic storm, resistance to atomic oxygen and radiation, etc.). The mass and harsh environment conditions constrain the design in that multiple dielectric layers are prohibited and only one metallic layer is advisable due to heat dissipation issues.

The most classical approach for designing a radar absorbing structure is the Salisbury screen [3], which consists in a resistive sheet mounted a quarter wavelength apart from a ground plane. This solution can be lightweight and has the advantage of simplicity. The Jauman absorber [4] generalizes this principle to multiple resistive layers in order to broaden the frequency band of absorption. This involves thickening and mass augmentation of the structure, which is not desirable for space application. A classical way to achieve a more versatile design is to replace the resistive sheets of Salisbury or Jauman absorbers by a lossy frequency selective surface (FSS). This can lead to a more compact design, like in the case of metamaterial absorbers [5], or to broadband solutions, like in circuit 
analog absorbers [6]. We will see that the presence of the FSS is also well-suited for oblique incidence as it provides additional parameters for the designer to use.

Many studies have been carried out concerning the design of wide-angle absorbers in the literature [7-10]. However, for all these designs, the maximum absorption is obtained for normal incidence and the performances degrade as the incidence angles increase. Article [11] is one of the first articles to address the question of oblique incidence in the literature, but the proposed design requires the use of relatively high permittivity (and high mass) dielectric substrates, which makes them unsuitable for space applications. We should also note that measurements of highly oblique angle of incidence are challenging [12].

In a previous study by the authors, two metamaterial absorbers for space applications based on resistive high impedance surface solution were proposed for the TT\&C (Telemetry, Tracking and Control) S-band [2 GHz, $2.3 \mathrm{GHz}$, optimized respectively for normal and oblique ( $60^{\circ}$ from normal direction) for both TE (Transverse Electric) and TM (Transverse Magnetic) polarization $[13,14]$ with experimental validation. These two metamaterial absorbers were designed for a specific and limited range of angle of incidence which was well adapted to the case of an antenna being positioned far away from the structure. However, when the antenna is close, it illuminates the structure over a wide angular range. The design of wide-angle absorbers centered on oblique incidence reveals to be a very challenging task. For a concrete scenario where antennas are mounted on satellite platforms, the position of the antennas is known in advance, which circumvents the problem. Instead of an ultra-wide-angle behavior, it should be enough to cover the platforms with different sectors, each being optimized for a specific angle of incidence in accordance with the antenna positions. This concept of an absorbing panel divided in different regions was first proposed in [15] and has been investigated numerically in [16].

In the current article, a sectorial absorber for the angular range $\left[35^{\circ}, 65^{\circ}\right]$ comprising two different sectors is designed. In the analysis, particular attention is paid to the angular behavior of the absorber. The sectorial absorber is fabricated and measured. A specific measurement setup is proposed to validate the concept of a sectorial absorber.

\section{Design}

In this section, we first specify the targeted performances, then describe the geometry of the absorber and we design two different sector absorbers consecutively. The angular behavior of the absorbers is emphasized and the difficulties concerning the design of wide angular range absorbers are discussed.

\subsection{Sectorial Absorber Concept and Objective}

For satellites, the emitting source is positioned close to the platform at a position which is known in advance (Figure 1). The purpose of the absorber is to limit multipath effects which could lead to interferences with other devices positioned on the platform. The close position of the source provides an angle of incidence which is oblique and that covers a large angular range. In this scenario, the common way to design absorbers with optimization at normal incidence is not appropriate. Thus, this article focuses on oblique incidence with $\theta$ varying from $35^{\circ}$ to $65^{\circ}$. For this range, absorption of $13 \mathrm{~dB}$ in the frequency band [ $2 \mathrm{GHz}, 2.3 \mathrm{GHz}]$ is set as objective.

This kind of performances cannot be achieved with a single low mass planar absorber. The absorbing surface is thus divided into different regions with respect to the incoming angle, as represented in Figure 1. For each region, the dimensions of the unit cell are optimized separately for a specific and narrower range of angle of incidence. The design of each sector thus becomes relatively simple and can be addressed using low mass structures and well-known FSS patterns like rectangular patches. The final step is to join and combine these different sectors together to increase the absorbing range of incidence of the whole structure. 


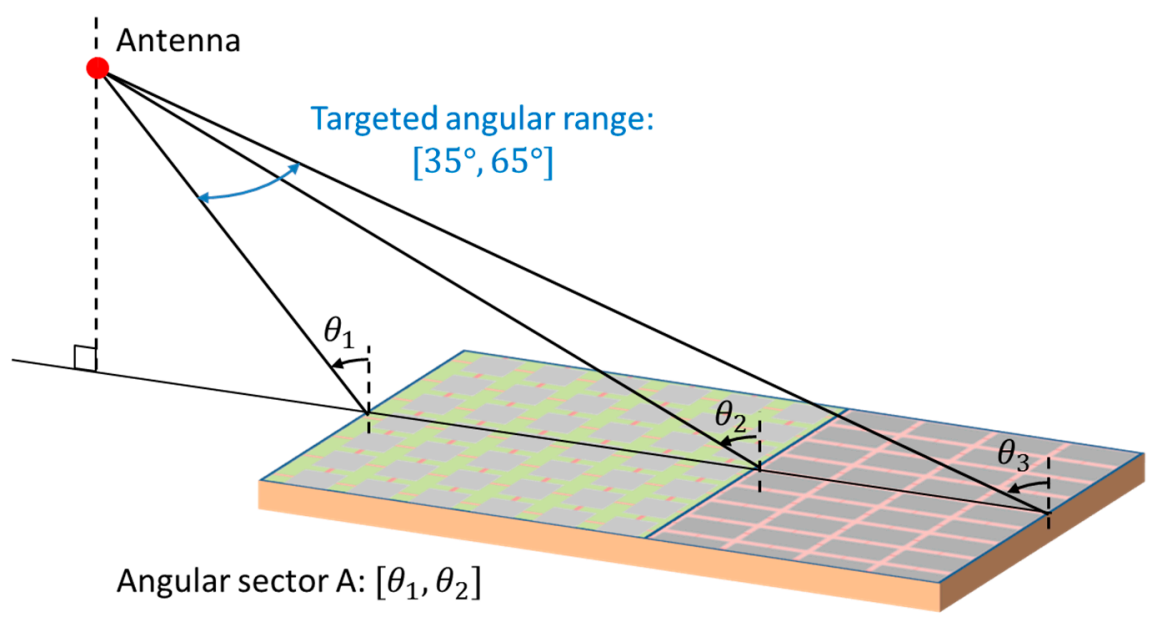

Angular sector $\mathrm{B}:\left[\theta_{2}, \theta_{3}\right]$

Figure 1. Angular range targeted for the present study. The platform is separated in two sectors for which the absorber can be optimized on a narrower range of incident angles.

\subsection{Description of the Absorbers}

The base case absorber (Figure 2a) is composed of a resistive FSS mounted at a distance of approximately $\lambda / 4$ from a copper ground plane. They are separated by a low mass dielectric substrate (Nomex Honeycomb) and the FSS is covered with a thin dielectric layer (RO4003C), which serves both as a mechanical support and as protection. The FSS is a periodic structure (Figure 2b) comprising rectangular metallic patches connected by resistive strips made of a TCR foil of $100 \Omega / \square$.

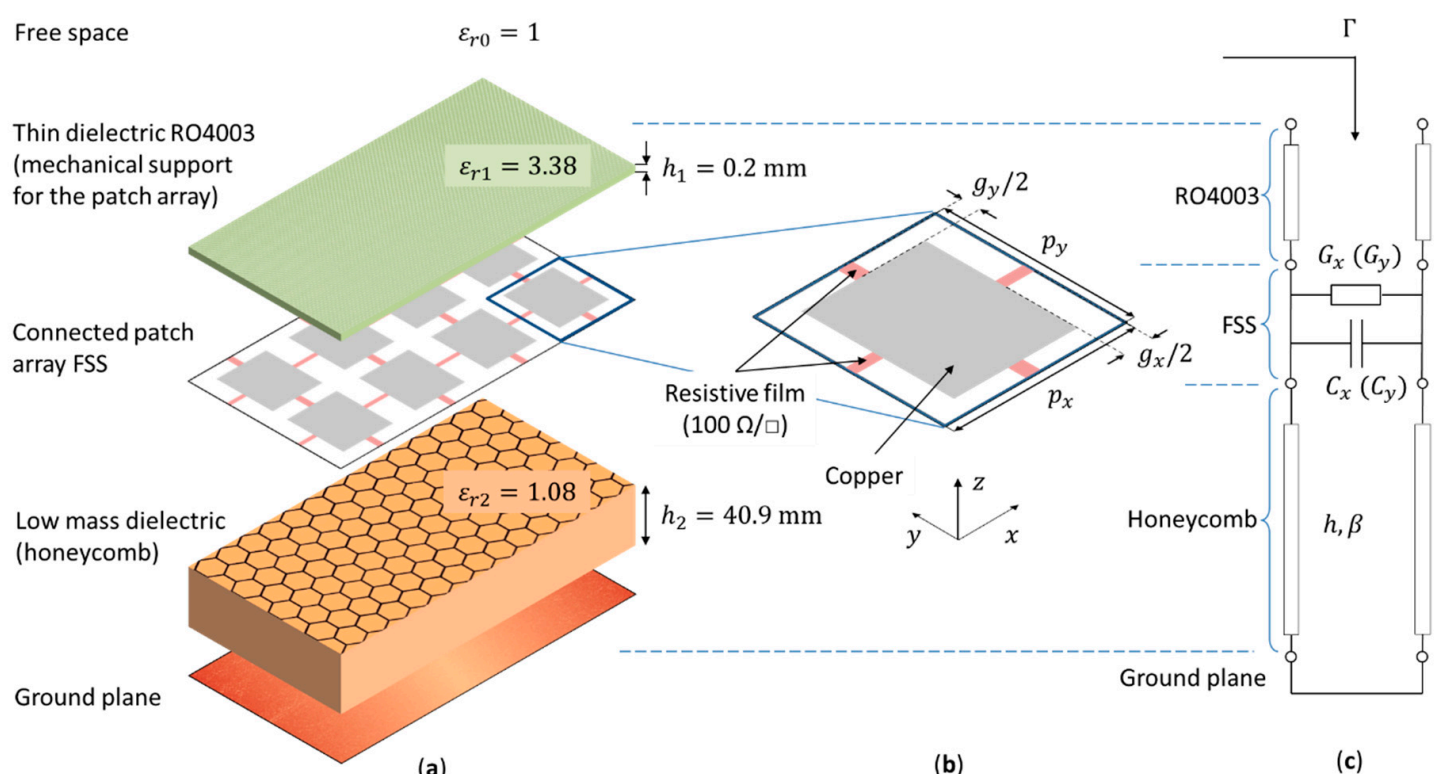

Figure 2. Geometry of the absorber (a) and of the rectangular unit cell of the frequency selective surface (FSS) (b). Dimensions along $x$ and y directions are dedicated to TE and TM polarizations respectively. (c) Equivalent circuit valid for each polarization.

An equivalent electric model (Figure 2c) is established for fast calculation. The dielectric layers are assimilated to transmission line sections and the FSS is described by its equivalent admittance [17] ( $G, C$ parallel circuit). The conductance $G$ is determined by the surface resistance $R_{s}$ of the TCR foil and the dimensions of the resistive strips. The shunt capacitance $C$ is related to the periodicity $p$, to the 
gap $g$, and to the dielectric permittivity of the surrounding layers. As each layer contribution is in parallel in the model (Figure 2c), it is convenient to work with admittance.

For an oblique angle of incidence $\theta$, the admittance of the incoming wave depends on the polarization as:

$$
\begin{gathered}
Y_{0}^{T E}=Y_{0} \cdot \cos \theta \\
Y_{0}^{T M}=Y_{0} / \cos \theta
\end{gathered}
$$

where $Y_{0}$ is the free space admittance. From transmission line theory, the reflection coefficient at the interface $\Gamma$ is given by

$$
\begin{aligned}
\Gamma^{T E} & =\frac{Y_{0} \cdot \cos \theta-Y_{i n}}{Y_{0} \cdot \cos \theta+Y_{i n}}, \\
\Gamma^{T M} & =\frac{Y_{0} / \cos \theta-Y_{\text {in }}}{Y_{0} / \cos \theta+Y_{\text {in }}},
\end{aligned}
$$

where $Y_{i n}$ is the input admittance of the absorber. This leads to a seemingly contradictory problem when trying to minimize the reflection coefficient for both polarizations simultaneously. To tackle this problem, a practical solution proposed in [14] is to consider an FSS with different dimensions along $x$ and $y$ directions, each one dedicated to one polarization. This leads to a rectangular unit cell, as shown in Figure $2 \mathrm{~b}$. Note that for this approach the wave vector has to be comprised in the $x z$ plane (i.e., $\varphi=0)$.

For optimization, the transmission line (TL) modeling approach [13-17] is used. It is however possible to derive a simple analytical model using two basic approximations. First, we assimilate the honeycomb $\left(\varepsilon_{r}=1.08\right)$ to free space. Second, due to its small thickness $\left(h_{1}=0.2 \mathrm{~mm}\right)$, the admittance of the RO4003C dielectric sheet is regarded as a shunt capacitance for which the value for normal incidence is given [18] by $C_{1}=Y_{0} \frac{\left(\varepsilon_{r 1}-1\right) \cdot h_{1}}{c}$. For oblique incidence, the effective value $C_{1}$ also depends on incidence $\theta$ and on polarization [18]. Considering the equivalent circuit of Figure 2c, $Y_{i n}$ is then given by

$$
\begin{gathered}
Y_{i n}{ }^{T E}=G+j\left[\omega C_{1} \cdot \cos \theta+\omega C \cdot\left(1-\varepsilon_{f} \cdot \sin 2 \theta\right)-Y_{0} \cdot \cos \theta \cdot \cot \left(\beta \cdot h_{2} \cdot \cos \theta\right)\right], \\
Y_{i n}{ }^{T M}=G+j\left[\omega C_{1} / \cos \theta+\omega C-Y_{0} / \cos \theta \cdot \cot \left(\beta \cdot h_{2} \cdot \cos \theta\right)\right],
\end{gathered}
$$

where $\varepsilon_{f}$ is the effective permittivity of the FSS calculated with the help of [19]. The maximum absorption is obtained when $Y_{\text {in }}$ is matched with the admittance of the incoming wave Equations (3) and (4). This appends at the resonance $\left[\operatorname{Im}\left(Y_{\text {in }}\right)=0\right]$ when $G$ equals $Y_{0}^{T E, T M}$.

\subsection{Optimization of Sector A}

A first optimization is conducted for one single absorber sector using the TL model and the genetic algorithm of Matlab. The optimization results are presented in Figure 3 and the corresponding values of the geometric parameters are provided in Table 1. The Matlab results are compared against full wave simulations (CST Microwave Studio, F solver with Floquet boundaries). There is a very good agreement, which validates the model.

Table 1. Geometrical dimensions of sector A for each polarization.

\begin{tabular}{ccccc}
\hline & $\mathbf{p}(\mathbf{m m})$ & $\mathbf{g}(\mathbf{m m})$ & $\mathbf{w}(\mathbf{m m})$ & $\mathbf{Z}(\Omega)$ \\
\hline TE $(\mathrm{x})$ & 5.1 & 1.6 & 0.32 & 500 \\
TM $(\mathrm{y})$ & 5 & 1 & 0.4 & 250 \\
\hline
\end{tabular}




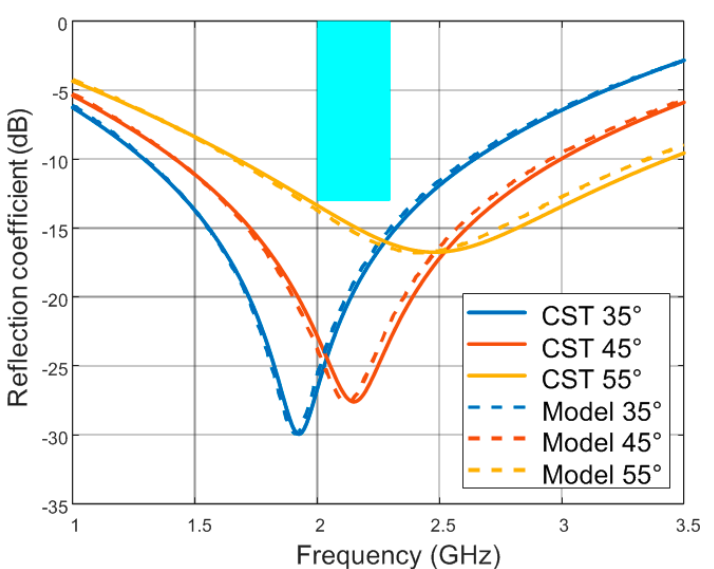

(a)

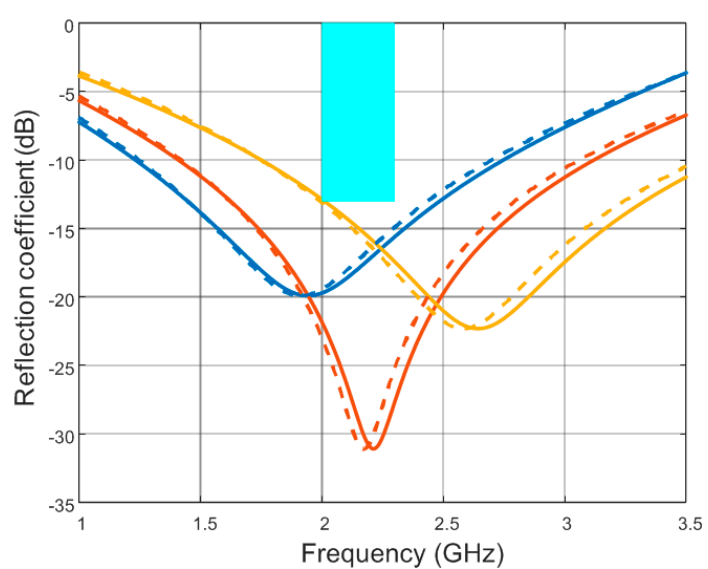

(b)

Figure 3. Comparison between TL model and EM simulation of sector A for TE polarization (a) and TM polarization (b). The rectangle corresponds to an objective of $-13 \mathrm{~dB}$ in the band [ $\mathrm{GHz}, 2.3 \mathrm{GHz}$.

The optimization results presented in Figure 3 show that the desired absorption level $(-13 \mathrm{~dB})$ is achieved only for a limited range of angle of incidence corresponding to $\left[35^{\circ}, 55^{\circ}\right]$ for both TE and TM polarizations. This limitation is due to two phenomena typical from oblique incidence:

- The resonant frequency, for which the reflection is minimum, shifts toward high frequency for increasing angles. For TE (Figure 3a), we observed a shift from $1.9 \mathrm{GHz}$ to $2.5 \mathrm{GHz}$ for $35^{\circ}$ and $55^{\circ}$ respectively.

- The absorption level varies with $\theta$. For TE, we observe that the absorption ranges from $-30 \mathrm{~dB}$ to $-15 \mathrm{~dB}$ for $35^{\circ}$ and $55^{\circ}$, respectively.

These two phenomena can be understood by inspecting the evolution of $Y_{\text {in }}$ with respect to $\theta$ at the fixed frequency of $2.15 \mathrm{GHz}$ (Figure 4).

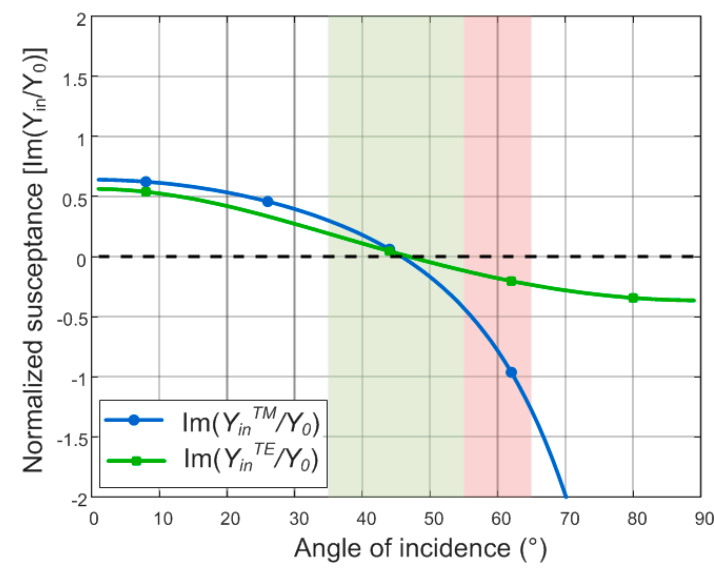

(a)

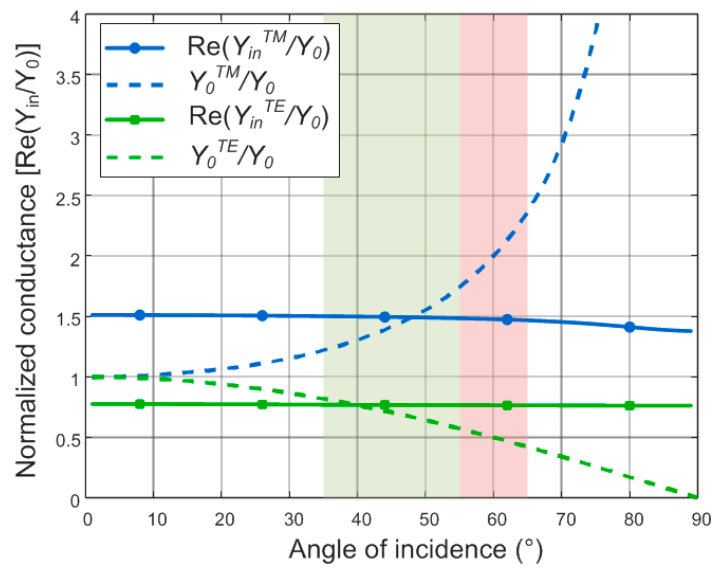

(b)

Figure 4. Imaginary (a) and real (b) part of $Y_{i n} / Y_{0}$ with respect to the angle of incidence at $2.15 \mathrm{GHz}$ for sector A and both polarizations.

As represented in Figure 4a, the imaginary part of $Y_{\text {in }}$ varies with $\theta$. At $2.15 \mathrm{GHz}$, we see that the dimensions of sector $\mathrm{A}$ are chosen such that the imaginary part of $Y_{\text {in }}$ cancels out at $45^{\circ}$ for both TE and TM polarizations. We know, from Equations (5) and (6), that $\operatorname{Im}\left(Y_{i n}\right)$ depends both on $\theta$ and on frequency, which explains the scan behavior observed in Figure 3a. The different shapes of the curves observed for TE and TM polarizations in Figure 4a are mainly due to the last term of Equations (5) and (6), which is directly related to the stub (honeycomb + ground plane). For TE, the variation of the 
admittance $\left(Y_{0} \cdot \cos \theta\right)$ compensates the cotangent term such that $\operatorname{Im}\left(Y_{\text {in }}\right)$ remains bounded for grazing incidence. This is not the case for TM, for which the variation rate increases with $\theta\left(\Delta Y_{i n}{ }^{T M}=0.3 Y_{0}\right.$ for $\left[0^{\circ}, 35^{\circ}\right], 0.7 Y_{0}$ for $\left[35^{\circ}, 55^{\circ}\right]$, and $0.9 Y_{0}$ for $\left.\left[55^{\circ}, 65^{\circ}\right]\right)$. This evolution illustrates the difficulty of designing wide-angle absorbers for high oblique incidence.

The real part of $Y_{\text {in }}$ (Figure $4 \mathrm{~b}$ ) is relatively constant with respect to $\theta$ but needs to be matched with the admittance of the incoming wave, which varies according to Equations (1) and (2). This explains the second point, as when $\theta$ varies, this directly affects the mismatch according to Equations (3) and (4), resulting in a lower absorption level. Interestingly, the cancelling out condition $G=Y_{0}^{T E}, T M$ is not chosen at resonance $\left(40^{\circ}\right.$ for TE and $48^{\circ}$ for TM instead of being at exactly $\left.45^{\circ}\right)$, which allows the widening of the frequency bandwidth at the expense of the minimum reflection level [20]. Similarly to what is observed for the imaginary part, the variation rate of $Y_{0}^{T E}$ and $Y_{0}^{T M}$ increases with $\theta$. This is more pronounced for $\mathrm{TM}$, for which $\Delta Y_{0}^{T M}=0.2 Y_{0}$ for $\left[0^{\circ}, 35^{\circ}\right], 0.5 Y_{0}$ for $\left[35^{\circ}, 55^{\circ}\right]$, and $0.6 Y_{0}$ for $\left[55^{\circ}, 65^{\circ}\right]$.

A classical method allowing both scan compensation and bandwidth enhancement is to use dielectric materials with higher permittivity instead of the honeycomb and to add a dielectric matching slab of approximately $\lambda / 4$ in front of the absorber [7]. This solution results in a consequent mass augmentation and is thus not adapted to space conditions. Another approach which is used in the study is to adjust the value of $C$ and $G$ in Equations (5) and (6) in order to obtain the desired frequency and absorption level for a given value of $\theta$. This does not compensate for the scan and mismatch issues when $\theta$ varies but allows a locally satisfactory solution. In practice, this amounts to modifying the geometric dimensions of the unit cell and therefore to designing separate sectors adapted to a more limited angular range.

\subsection{Optimization of Sector B}

A second absorber, denoted sector $\mathrm{B}$, is optimized using a genetic algorithm to operate on the angular range $\left[55^{\circ}, 65^{\circ}\right]$. For facilitating the manufacture, the honeycomb thickness is chosen constant for all sectors. The optimization thus only affects the geometrical parameters of the FSS. The optimizations results are presented in Figure 5 and the corresponding values of the geometric parameters are given in Table 2.

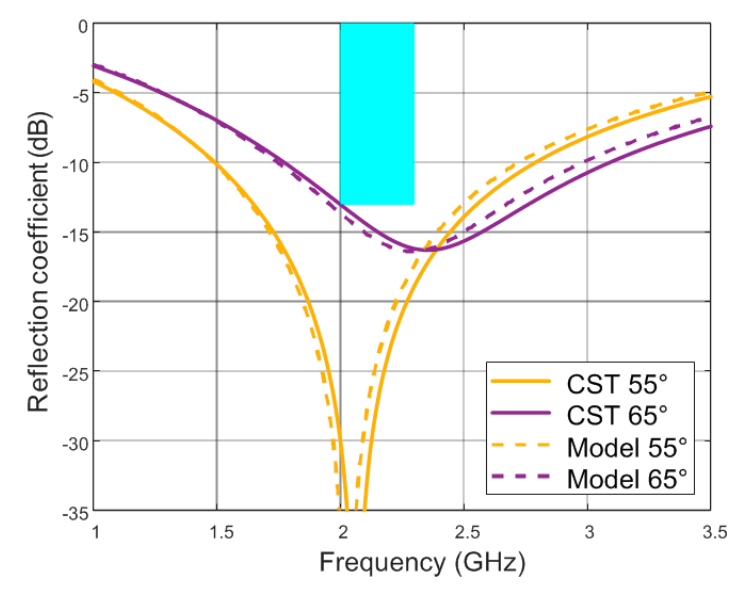

(a)

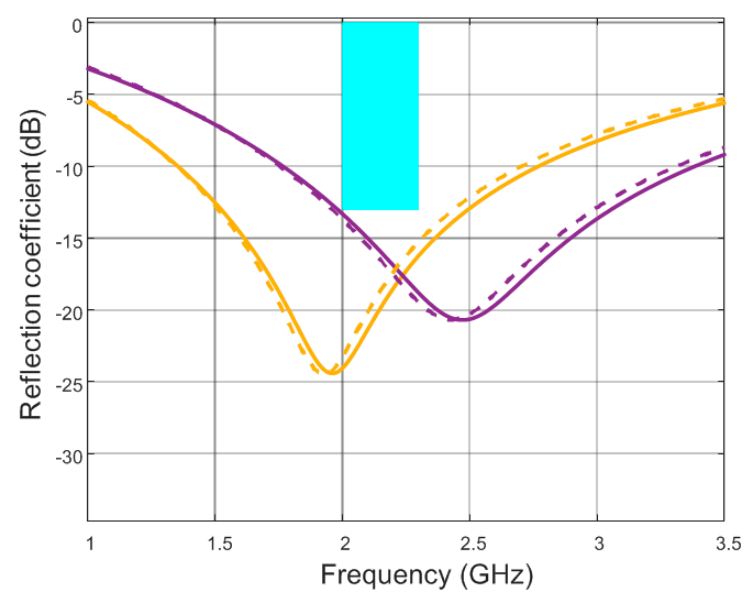

(b)

Figure 5. Comparison between analytical model and EM simulation of sectorial absorber B for TE polarization (a) and TM polarization (b). 
Table 2. Geometrical dimensions of the sectorial absorber B for each polarization.

\begin{tabular}{ccccc}
\hline & $\mathbf{p}(\mathbf{m m})$ & $\mathbf{g}(\mathbf{m m})$ & $\mathbf{w}(\mathbf{m m})$ & $\mathbf{Z}(\Omega)$ \\
\hline TE $(\mathrm{x})$ & 10.5 & 2 & 0.45 & 444 \\
$\mathrm{TM}(\mathrm{y})$ & 15.5 & 1.3 & 0.46 & 283 \\
\hline
\end{tabular}

Similarly to what is observed for sector A, a good agreement between simulation and model is obtained. A maximum frequency shift of $50 \mathrm{MHz}$ is observed for $65^{\circ}$ in TE, which is probably due to the fact that the model does not take into account high order resonant modes of the FSS. As with sector A, we observe a scan and a mismatch effect, which limits the operational angular range of the design. For both polarizations, the resonance condition at $2.15 \mathrm{GHz}$ is set at $60^{\circ}$ (center of the targeted incidence range). For this frequency, $G$ is matched to $Y_{0}^{T E}$ at $55^{\circ}$ for TE and to $Y_{0}^{T M}$ at $60^{\circ}$ for TM. The absorber complies with the requirement of $-13 \mathrm{~dB}$ between $2 \mathrm{GHz}$ and $2.3 \mathrm{GHz}$ (represented by a blue rectangle in the graph).

Finally, the reflection coefficient with respect to incident angle is represented at $2.15 \mathrm{GHz}$ for sectors $A$ and $B$ and both polarizations in Figure 6 . We observe that both sectors are operating in the targeted angular range. Sector $A$ has a reflection coefficient inferior of $-15 \mathrm{~dB}$ in $\left[35^{\circ}, 55^{\circ}\right]$ and sector $B$, a reflection coefficient inferior of $-17 \mathrm{~dB}$ in $\left[55^{\circ}-65^{\circ}\right]$. The sector $\mathrm{A}$ remains below $-10 \mathrm{~dB}$ from normal incidence to $60^{\circ}$. It is interesting to note that in Figure 6, the absorption peaks with respect to angle are more selective for TM polarization than for TE, which is consistent with the angular sensitivity analysis made for Figure 4.

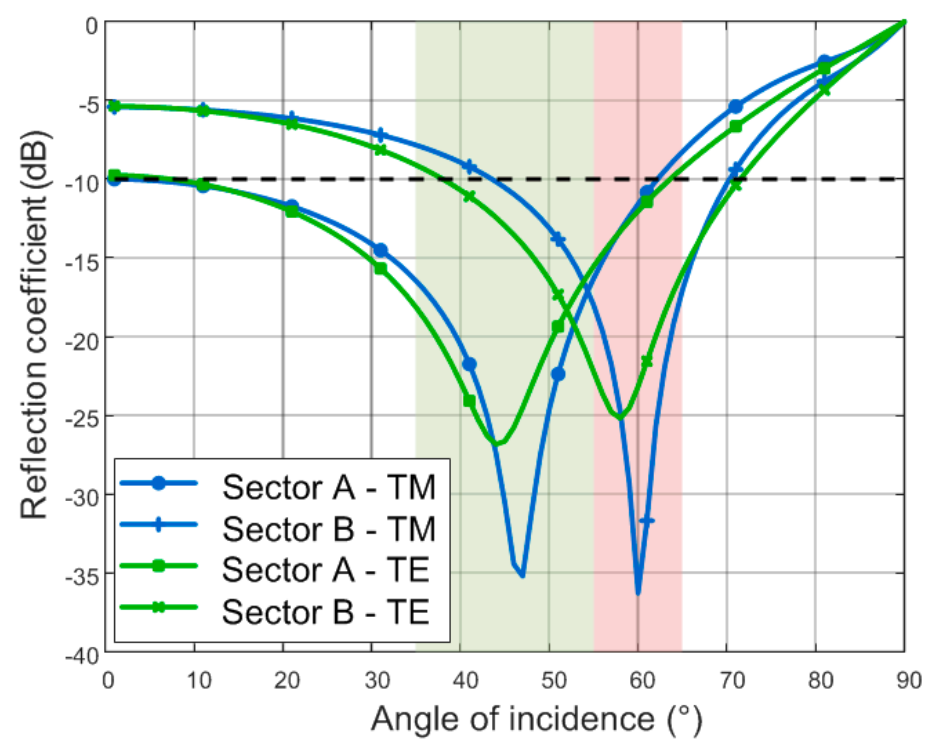

Figure 6. Reflection coefficient of sectorial absorbers A and B for both polarizations with respect to angle of incidence. TL model, $2.15 \mathrm{GHz}$.

\section{Measurement}

\subsection{Prototypes}

Two panels were realized for each sector. The two panels corresponding to sector A are shown in Figure 7a. The FSSs (Figure 7b) were realized by CIRETEC using two etching sequences. The copper foil and the FSS layer were then attached to the honeycomb using double-sided adhesive film for a total thickness of $41.1 \mathrm{~mm}$. The total size of each prototype is $578 \mathrm{~mm} \times 428 \mathrm{~mm}$ (Figure 7c) which is approximately $3.9 \lambda \times 2.9 \lambda$ at $2 \mathrm{GHz}$. For the sectorial absorber, two panels corresponding to sector $\mathrm{A}$ and a sector $B$ were positioned side by side, resulting in a $1156 \mathrm{~mm} \times 428 \mathrm{~mm}$ structure. The sectorial absorber composed of a panel $\mathrm{A}$ adjoined to a panel $\mathrm{B}$ is denoted by 'A $+\mathrm{B}$ ' in the rest of the article. 
In order to compare the performances of the 'A $+\mathrm{B}$ ' absorber with the uniform absorbers of the same dimensions, two other configurations were considered corresponding to panel $A$ adjoined to a second panel A (denoted by 'A $+A^{\prime}$ ) and panel B adjoined to a second panel B (denoted by 'B $+B^{\prime}$ ).

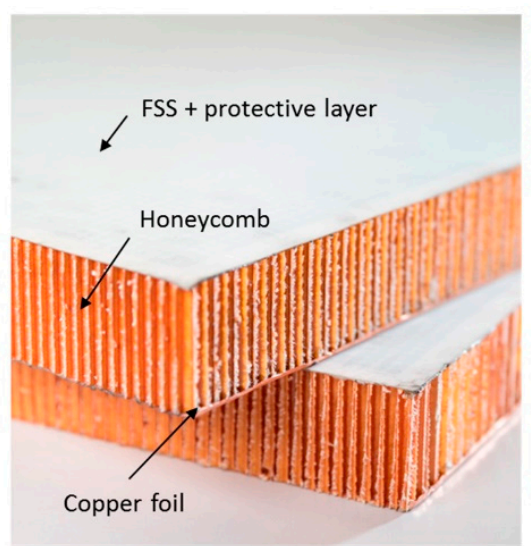

(a)

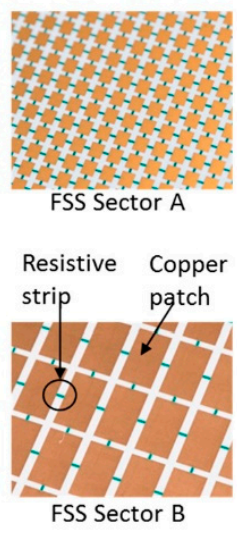

(b)

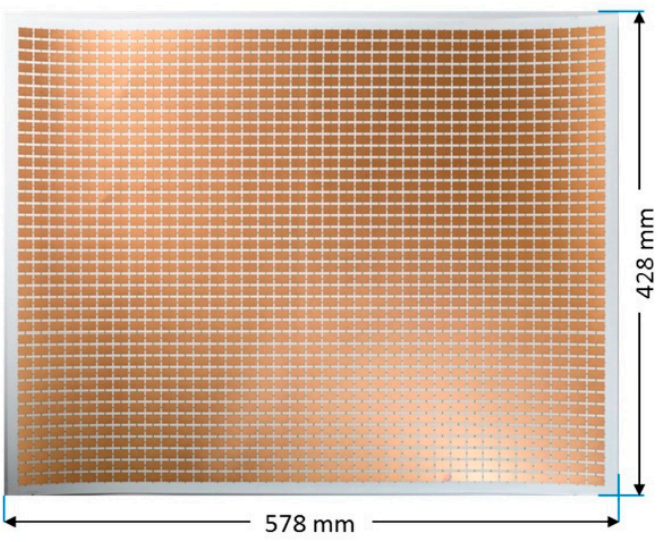

(c)

Figure 7. Pictures of the manufactured prototypes. (a) Two panels of sector A absorber. (b) FSS patterns of sector A and sector B. (c) Single panel dimensions.

\subsection{Measurement Setup}

The reflection coefficients of the absorbers were measured using free space (planar wave) method [21] in a bistatic configuration. The setup is presented schematically Figure 8 and a picture is given in Figure 9. In order to be representative of the application (antenna on platform) the emitting antenna was static and directed at the device under test such that that sectors A and B were illuminated with the proper range of incidence angles. For each angle of interest, the receiving antenna was mounted at one meter from the corresponding reflection point and directed toward the specular angle, as represented in Figure 8.

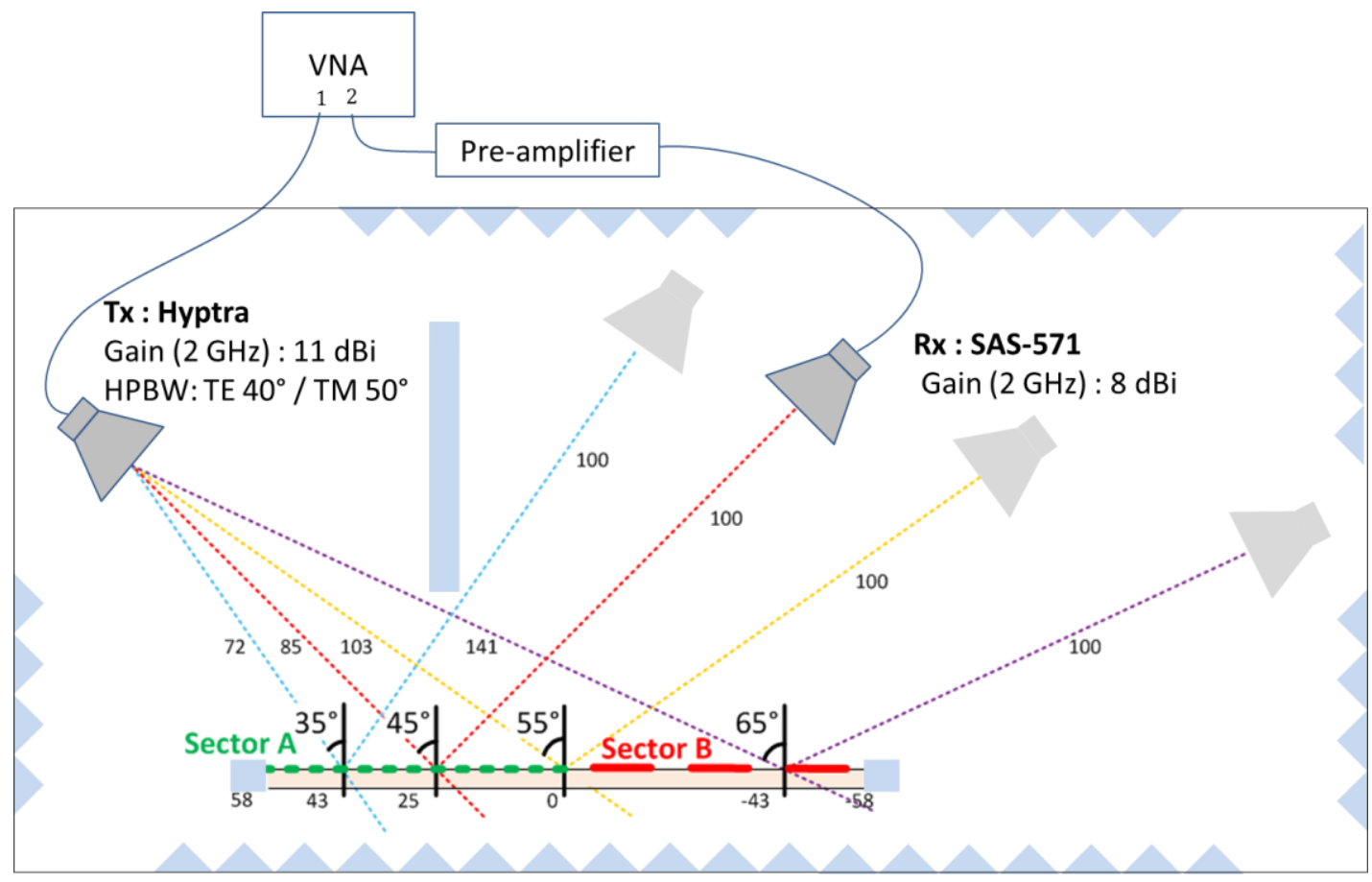

Figure 8. Bistatic measurement setup in anechoic chamber. Each sector was illuminated with the proper angle range. The Tx antenna was static while the Rx antenna was moved for each angle of interest. 


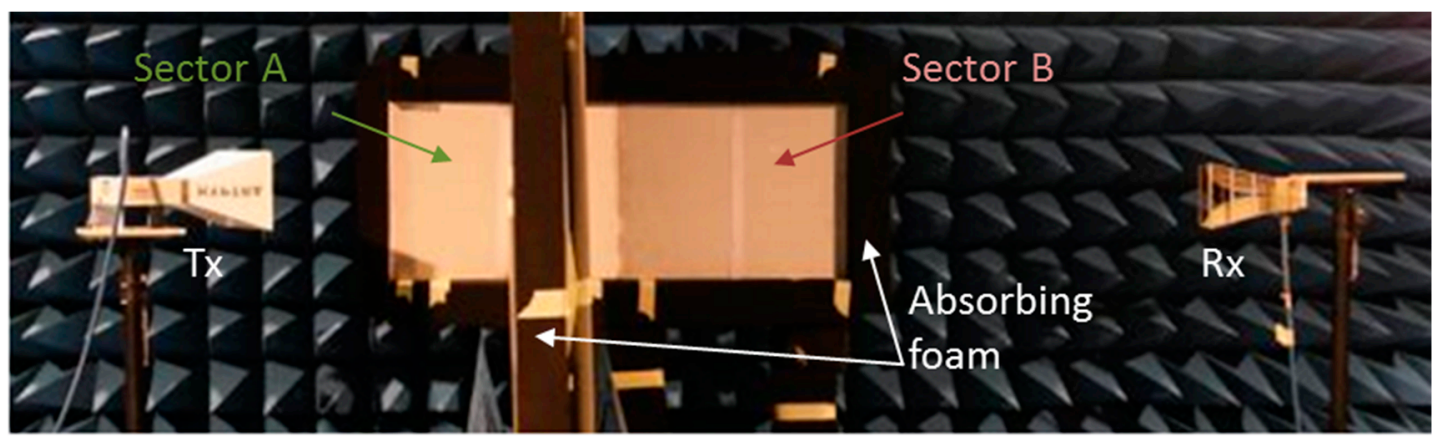

Figure 9. Picture of the measurement setup.

Measurements were performed in an anechoic chamber (Figure 9). Tx and Rx antennas were separated by absorbing foam to limit direct crosstalk. Additional foam was placed at the wedge of the sectorial absorbers to limit both the wedge diffraction and excitation of surface waves for oblique incidence. The measurements were performed using Keysight E5071C VNA and a pre-amplifier in reception. The transmitting antenna was the Hyptra horn antenna which has a gain of $11 \mathrm{dBi}$ and a half power beam width (HPBW) of $40^{\circ}$ and $50^{\circ}$ for $\mathrm{E}$ and $\mathrm{H}$ planes at $2 \mathrm{GHz}$. The receiving antenna was the commercial SAS-571 horn antenna, which has a gain of $8 \mathrm{dBi}$ at $2 \mathrm{GHz}$. The results were normalized with respect to a metallic plate of same size. In addition, time gating (Hann window) was applied to measured data in order to remove parasitic reflection from the setup.

\subsection{Measurement Results}

The sectorial absorber $\mathrm{A}+\mathrm{B}$ was measured for angles $35^{\circ}, 45^{\circ}, 55^{\circ}$ and $65^{\circ}$. For the sake of comparison, two other measurements corresponding to sector $\mathrm{A}+\mathrm{A}$ and sector $\mathrm{B}+\mathrm{B}$ were also conducted. The overall dimensions of sector $\mathrm{A}+\mathrm{B}, \mathrm{A}+\mathrm{A}$ and $\mathrm{B}+\mathrm{B}$ are the same. The measurement results are compared with each other and also against full-wave simulations (uniform infinite array, CST A and CST B) for each angle in Figure 10.

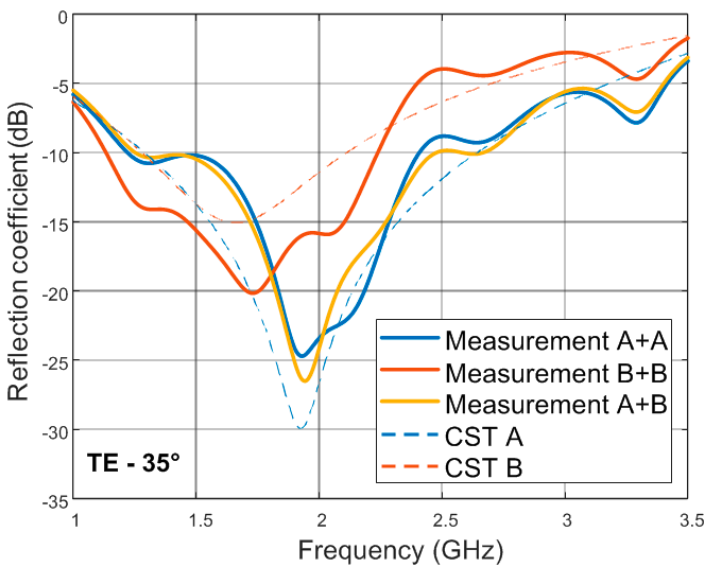

(a)

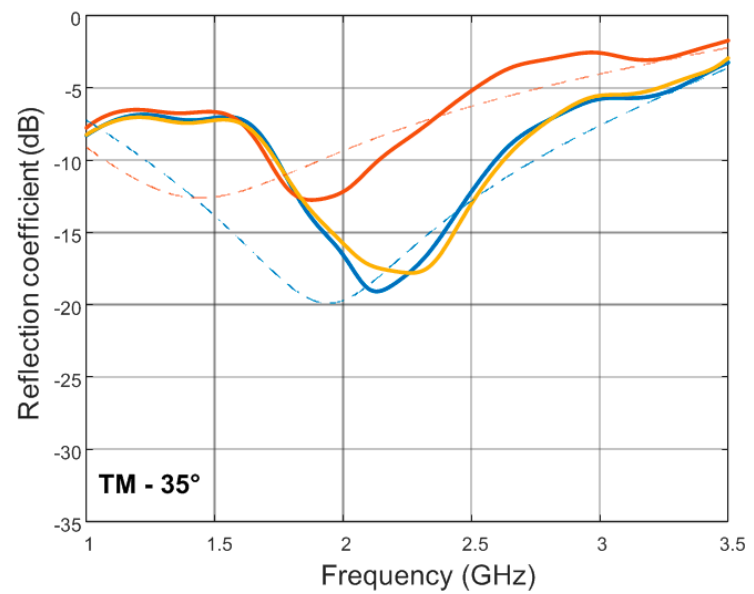

(b)

Figure 10. Cont. 


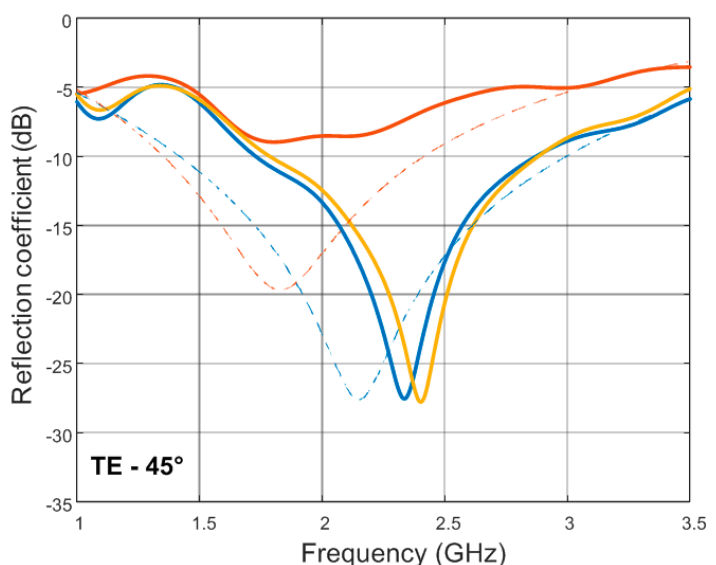

(c)

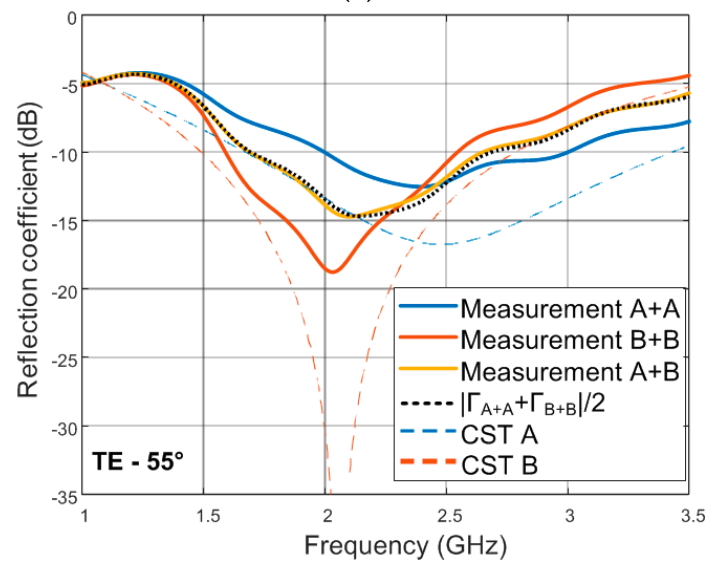

(e)

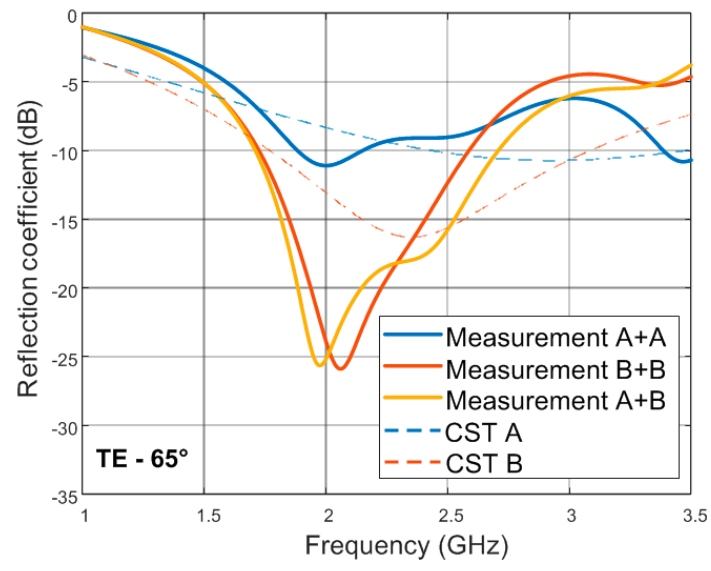

(g)

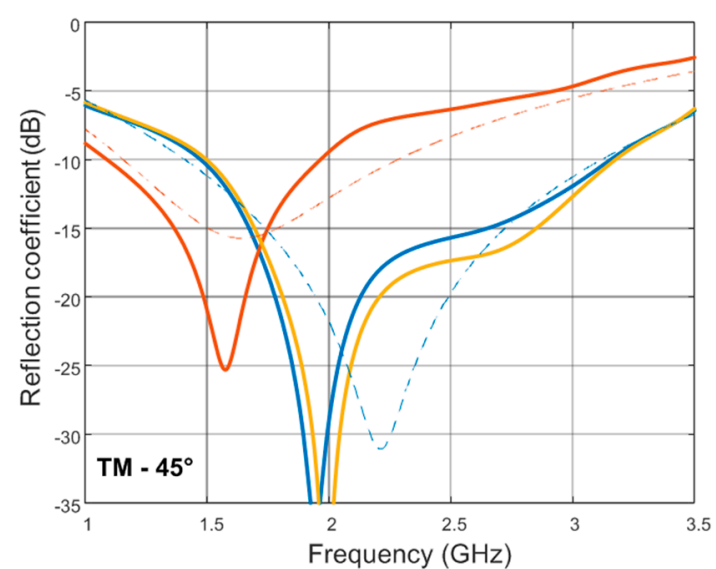

(d)

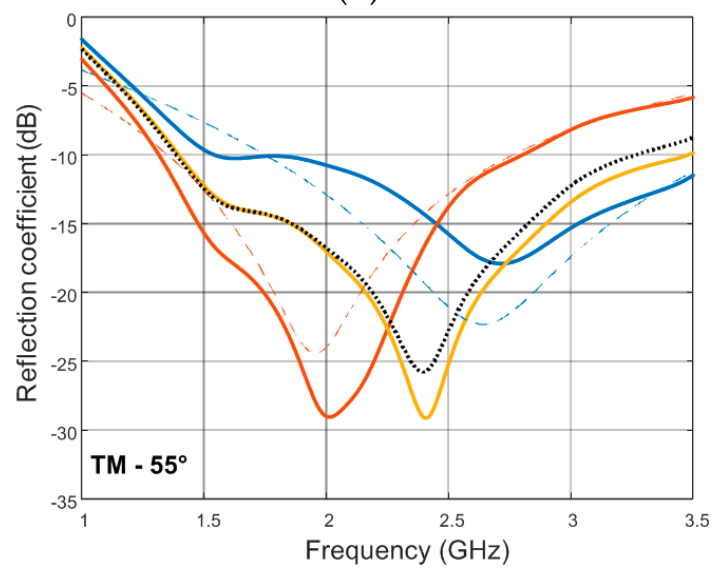

$(\mathbf{f})$

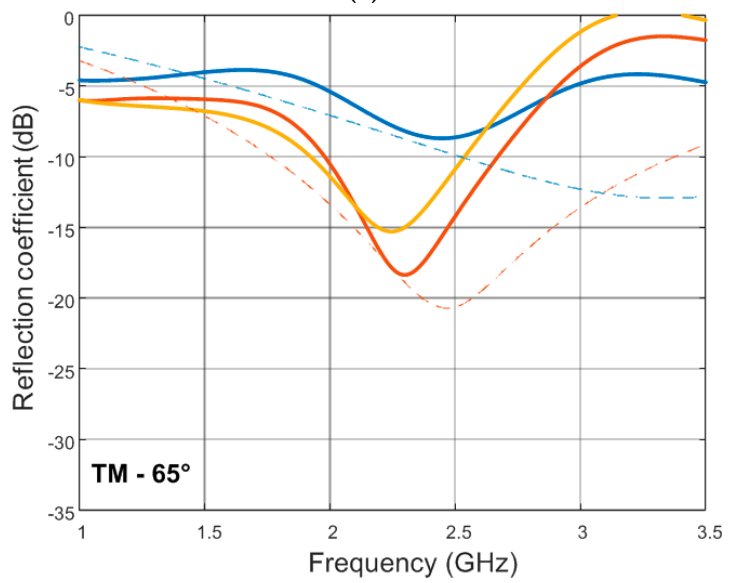

(h)

Figure 10. Bistatic measurement of the sectorial absorber for TE polarization at $35^{\circ}$ (a), $45^{\circ}$ (c), $55^{\circ}$ (e) and $65^{\circ}(\mathrm{g})$ and TM polarization at $35^{\circ}(\mathbf{b}), 45^{\circ}(\mathbf{d}), 55^{\circ}(\mathbf{f})$ and $65^{\circ}(\mathbf{h})$. The measurements of sectorial absorber $\mathrm{A}+\mathrm{B}$ is compared against uniform absorbers $\mathrm{A}+\mathrm{A}$ and $\mathrm{B}+\mathrm{B}$ and also with the simulation of uniform infinite absorbers $\mathrm{A}$ and $\mathrm{B}$.

At $35^{\circ}$ (Figure 10a,b), the dips are well marked and the result of sector $A+B$ is very close to the one of the uniform sector A + A. As desired, sector A exhibits a better absorption than sector B for this angle. For TE, the frequency positions of the dips are in good agreement with simulations for both uniform absorbers. For TM, the absorption levels are well respected between simulation and measurement but the measurements are more selective and there is a frequency shift of approximately 
$250 \mathrm{MHz}$ for uniform absorber A + A. The absorption band remains well positioned relatively to the [2 GHz, 2.3 GHz] objective.

At $45^{\circ}$ (Figure 10c,d), the curves are smoother than for other measurements, which could be explained by the fact that the reflection point is located in the middle of the panel, relatively far from wedges and from the transition between the two panels (see Figure 8). Like before, the curve of sector $A+B$ is very close to sector $A+A$ and sector $A$ exhibits a higher absorption than sector $B$ for both polarizations. The measurements show more selective dips than the simulations. For TE, there is an acceptable agreement between measurement and simulation for uniform sector A despite a frequency shift of approximately $300 \mathrm{MHz}$. Sector B measurements are degraded compared to simulations. For $\mathrm{TM}$, a shift of approximately $-300 \mathrm{MHz}$ is observed for sector $\mathrm{A}+\mathrm{A}$ and sector $\mathrm{A}+\mathrm{B}$.

At $55^{\circ}$ (Figure 10e,f), the reflection point corresponds exactly to the transition between the two panels. For sector $\mathrm{A}+\mathrm{B}$, the backscattered signal is the complex average between the $\mathrm{A}$ and $\mathrm{B}$ contributions. For comparison, the average between reflection coefficients measured for sectors $\mathrm{A}+\mathrm{A}$ and B $+\mathrm{B}\left(\left|\Gamma_{A+A}+\Gamma_{B+B}\right| / 2\right)$ is also reported with the dotted black lines in the graph. For TE, there is a very good agreement between the average and the measurement of sector $\mathrm{A}+\mathrm{B}$. For TM, we also observe a good agreement between the average and the measured sector $\mathrm{A}+\mathrm{B}$. In this case, $\mathrm{A}$ and $\mathrm{B}$ are out of phase $\left(\Delta \varphi=260^{\circ}\right.$ between $\mathrm{A}$ and $\mathrm{B}$ at $2.4 \mathrm{GHz}$ from measurements) which contributes to shift the dip of A + B compared to either A or B. Note that this kind of destructive interference between the backscattered field from adjacent pavings has been exploited in recent years to design metallic-only absorbers [22]. For TE, the comparison between measurement and simulation is acceptable despite a deviation in the level of the reflection coefficient for both sectors A and B. For TM, we also observe a discrepancy concerning the level, but the resonant frequencies are well respected.

At $65^{\circ}$ (Figure $10 \mathrm{~g}, \mathrm{~h}$ ), it is observed that, contrarily to $35^{\circ}$ and $45^{\circ}$, the sector $\mathrm{A}+\mathrm{B}$ becomes very close to $\mathrm{B}+\mathrm{B}$ for both polarizations. As expected from the design, the sector $\mathrm{B}$ shows higher absorption than sector $A$ at this angle. For both polarizations, the comparison with simulation is not particularly good, which can be explained by the fact that the contribution of the wedges becomes critical when approaching grazing incidence. Another possible explanation is that the simulations were carried out for infinite array, while the physical cross-section of the absorber becomes very thin compared to the wavelength for high angles, which accentuates the finitude effects. This last hypothesis seems to be in accordance with the fact that TE seems more degraded than TM, especially for sector A $+\mathrm{A}$.

In summary, the measurement results presented in Figure 10 are all in reasonable agreement with the full-wave infinite-array simulations. We clearly see that $A+B$ behaves similarly to $A+A$ for $35^{\circ}$ and $45^{\circ}$ and similarly to $\mathrm{B}+\mathrm{B}$ for $65^{\circ}$. At the junction between the two panels $\left(55^{\circ}\right), \mathrm{A}+\mathrm{B}$ is the complex average between $\mathrm{A}+\mathrm{A}$ and $\mathrm{B}+\mathrm{B}$. These measurement results validate the sectorial absorber approach.

In order to provide a synthetic view of the absorber performance, the measurements of sector $\mathrm{A}+\mathrm{B}$ are reported on the same graph for all the measured angles in Figure 11. The performances are slightly degraded in measure compared to simulations. A reflection coefficient inferior to $-11.5 \mathrm{~dB}$ (instead of $-13 \mathrm{~dB}$ ) is measured for all angles of interest in the band [2 GHz, 2.3 GHz]. 


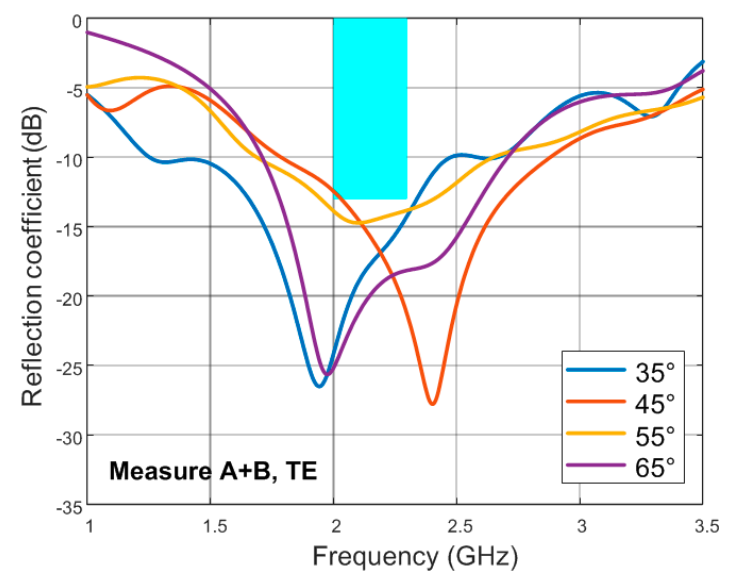

(a)

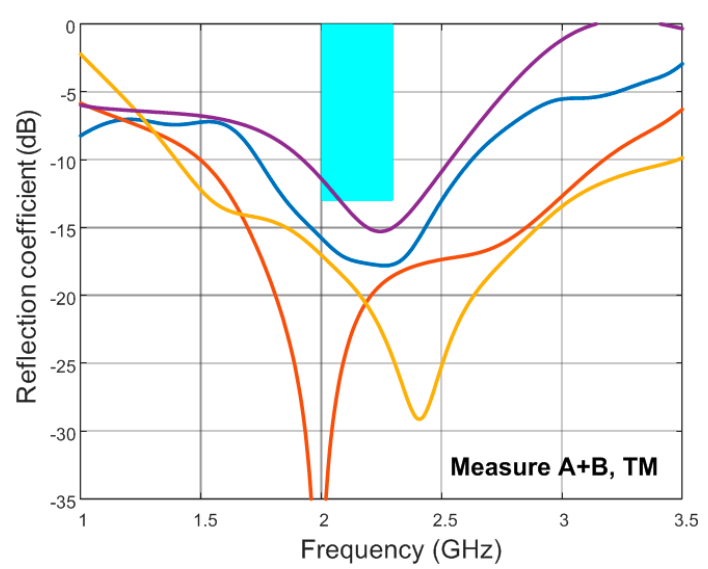

(b)

Figure 11. Bistatic measurement of the sectorial absorber $A+B$ at various angles for TE (a) and TM (b) polarizations. The rectangle features an objective of $-13 \mathrm{~dB}$ on the [2 GHz, $2.3 \mathrm{GHz}]$ band.

\section{Conclusions}

The design, realization and measurement of a thin lightweight absorbing material for space applications were presented. The behavior of the absorber with respect to incidence angle was emphasized during the design procedure and wide-angle performances for oblique incidence were shown to be particularly challenging due to increasing scan and mismatch phenomena. The problem was addressed by dividing the initial surface into two sectors, each being optimized for a narrower angular range. A specific measurement setup was proposed to show that the whole structure effectively showed performances expected by each separate sector when impinged with a proper incident angle. The measurements were performed in anechoic chamber. The absorber achieved a reflection coefficient inferior to $-11.5 \mathrm{~dB}$, corresponding to an absorptivity above 0.965 , over the $14 \%$ frequency band for [2 $\mathrm{GHz}, 2.3 \mathrm{GHz}$ ] for TE and TM polarizations for angles of incidence varying from $35^{\circ}$ to $65^{\circ}$.

The concept of a multi-sector absorber, demonstrated for two sectors in the current study, can be extended to more sectors in order to improve the performances. At the limit, the absorber could be continuously tapered in order to obtain a gradient FSS-absorber which would probably lead to better performances, although at the cost of simplicity.

Author Contributions: Concept and design, O.R. and A.C.L. and X.B.; Investigation, O.R and A.C.L; Simulations, O.R.; Measurement, O.R. and A.C.L.; Draft preparation, O.R. and A.C.L.; Review, X.B., K.E. and N.C.; Supervision, K.E.

Funding: This research was funded by the Centre National d'Etudes Spatiales (CNES).

Conflicts of Interest: The authors declare no conflict of interest.

\section{References}

1. Martel, C.; Gabard, B.; Boust, F.; Gobin, V.; Elis, K. A metamaterial electromagnetic wave absorber based on resistive films for micro-satellite spatial applications. In Proceedings of the 2018 IEEE International Symposium on Antennas and Propagation USNC/URSI National Radio Science Meeting, Boston, MA, USA, 8-13 July 2018; pp. 441-442.

2. Delfini, A.; Albano, M.; Vricella, A.; Santoni, F.; Rubini, G.; Pastore, R.; Marchetti, M. Advanced radar absorbing ceramic-based materials for multifunctional applications in space environment. Materials 2018, 11, 1730. [CrossRef] [PubMed]

3. Salisbury, W.W. Absorbent body for electromagnetic waves. U.S. Patent 2599944, 10 June 1952.

4. Toit, L.J.D. The design of Jauman absorbers. IEEE Antennas Propag. Mag. 1994, 36, 17-25. [CrossRef]

5. Engheta, N. Thin absorbing screens using metamaterial surfaces. In Proceedings of the IEEE Antennas and Propagation Society International Symposium, San Antonio, TX, USA, 16-21 June 2002; pp. 392-395.

6. Munk, B.A. Frequency Selective Surfaces-Theory and Design; Wiley-Blackwell: New York, NY, USA, 2000. 
7. Lee, D.; Hwang, J.G.; Lim, D.; Hara, T.; Lim, S. Incident angle- and polarization-insensitive metamaterial absorber using circular sectors. Sci. Rep. 2016, 6, 27155. [CrossRef] [PubMed]

8. Wang, J.; Yang, R.; Tian, J.; Chen, X.; Zhang, W. A dual-band absorber with wide-angle and polarization insensitivity. IEEE Antennas Wirel. Propag. Lett. 2018, 17, 1242-1246. [CrossRef]

9. Kalraiya, S.; Chaudhary, R.K.; Abdalla, M.A. Design and analysis of polarization independent conformal wideband metamaterial absorber using resistor loaded sector shaped resonators. J. Appl. Phys. 2019, 125, 134904. [CrossRef]

10. Nguyen, T.T.; Lim, S. Wide incidence angle-insensitive metamaterial absorber for both TE and TM polarization using eight-circular-sector. Sci. Rep. 2017, 7, 3204. [CrossRef] [PubMed]

11. Munk, B.A.; Munk, P.; Pryor, J. On designing jaumann and circuit analog absorbers (CA absorbers) for oblique angle of incidence. IEEE Trans. Antennas Propag. 2007, 55, 186-193. [CrossRef]

12. Alvarez, H.F.; de Cos Gomez, M.E.; Las-Heras, F. Angular stability of metasurfaces: challenges regarding reflectivity measurements. IEEE Antennas Propag. Mag. 2016, 58, 74-81. [CrossRef]

13. Pinto, Y.; Sarrazin, J.; Lepage, A.C.; Begaud, X.; Capet, N. Design and measurement of a thin and light absorbing material for space applications. App. Phys. A 2014, 115, 541-545. [CrossRef]

14. Pinto, Y.; Sarrazin, J.; Lepage, A.C.; Begaud, X.; Capet, N. Resistive high-impedance surfaces (RHIS) as absorbers for oblique incidence electromagnetic waves. Appl. Phys. A 2014, 117, 693-697. [CrossRef]

15. Capet, N.; Ballesteros, Y.C.P.; Lepage, A.C.; Sarrazin, J.; Begaud, X. Multi-Sector Absorbing Method and Device. U.S. Patent WO2015136121, 17 September 2015.

16. Lepage, A.C.; Pinto, Y.; Rance, O.; Begaud, X.; Capet, N. Lightweight and Wide-Angle Metamaterial Absorbing Material Concept. In Proceedings of the European Conference on Antennas and Propagation (EuCAP 2018), London, UK, 9-13 April 2018.

17. Luukkonen, O.; Simovski, C.; Granet, G.; Goussetis, G.; Lioubtchenko, D.; Raisanen, A.V.; Tretyakov, S.A. Simple and Accurate Analytical Model of Planar Grids and High-Impedance Surfaces Comprising Metal Strips or Patches. IEEE Trans. Antennas Propag. 2008, 56, 1624-1632. [CrossRef]

18. Magill, E.; Wheeler, H. Wide-angle impedance matching of a planar array antenna by a dielectric sheet. IEEE Trans. Antenn. Propag. 1966, 14, 49-53. [CrossRef]

19. Costa, F.; Monorchio, A.; Manara, G. Efficient Analysis of Frequency-Selective Surfaces by a Simple Equivalent-Circuit Model. IEEE Trans. Antennas Propag. 2012, 54, 35-48. [CrossRef]

20. Chambers, B. Optimum design of a Salisbury screen radar absorber. Electron.Lett. 1994, 30, $1353-1354$. [CrossRef]

21. Hiatt, R.E.; Knott, E.F.; Senior, T.B.A. A Study of VHF Absorbers and Anechoic Rooms; Report No. 5391-1-F; The University of Michigan/NASA, Langley Research Center: Hampton, VA, USA, 1963.

22. Paquay, M.; Iriarte, J.; Ederra, I.; Gonzalo, R.; Maagt, P. de Thin AMC structure for radar cross-section reduction. IEEE Trans. Antennas Propag. 2007, 55, 3630-3638. [CrossRef] 